\title{
Quantile regression with errors in variables ${ }^{\dagger}$
}

\author{
Jooyong Shim ${ }^{1}$ \\ ${ }^{1}$ Department of Data Science, Inje University \\ Received 4 February 2014, revised 16 February 2014, accepted 1 March 2014
}

\begin{abstract}
Quantile regression models with errors in variables have received a great deal of attention in the social and natural sciences. Some efforts have been devoted to develop effective estimation methods for such quantile regression models. In this paper we propose an orthogonal distance quantile regression model that effectively considers the errors on both input and response variables. The performance of the proposed method is evaluated through simulation studies.
\end{abstract}

Keywords: Check function, errors in variables, iteratively reweighted least squares procedure, orthogonal distance regression, quantile regression.

\section{Introduction}

Quantile regression (QR) introduced by Koenker and Bassett (1978) provides a more informative description of relationships between input variables and response variables. Just as classical linear regression methods based on minimizing sum of squared residuals enable us to estimate a wide variety of models for conditional mean functions, quantile regression methods offer a mechanism for estimating models for the full range of conditional quantile functions including the conditional median function. By supplementing the estimation of conditional mean functions with techniques for estimating an entire family of conditional quantile functions, quantile regression is capable of providing a better statistical analysis of the stochastic relationships among random variables. The introductions and applications of the quantile regression can be found in Koenker et al. (1994), Yu et al. (2003), Koenker (2005), Hwang (2010), Shim and Lee (2010), Lee (2012).

Most of this attention has been paid to data measured exactly without error but QR analysis with errors in variables (EIV) is evolving, albeit slowly. See, for example, Boggset al. (1987), Fuller (1987), He and Liang (2000), Chesher (2001), Schennach (2008), Hu and Schennach (2008), Ioannidesa and Matzner-L $\phi$ ber (2009), Wei and Carroll (2009), Ma and Yin (2011), Wang et al. (2012). However, less attention has been paid to QR with EIV than to mean regression with EIV because of two main difficulties for correcting the bias in QR caused by EIV (Wang et al., 2012). One is that a parametric regression-error likelihood is usually not specified in QR. The other is that the quantile of the sum of two random

\footnotetext{
$\dagger$ This research was supported by Basic Science Research Program through the National Research Foundation of Korea (NRF) funded by the Ministry of Education, Science and Technology (2012000646).

1 Adjunct professor, Institute of statistical Information, Department of Data Science, Inje University, Kimhae 621-749, Korea. E-mail: ds1631@hanmail.net
} 
variables is not necessarily the sum of the two marginal quantiles. In addition, most literature has centered around the parametric approach in which the quantile regression function is assumed to take on a particular functional form.

In this paper we consider an orthogonal distance quantile regression model (ODQR) with EIV by applying check function of orthogonal residuals to the formulation of quantile regression of Koenker and Basset (1978). Like He and Liang (2000), the proposed ODQR assumes that the random errors in the response variable and the measurement errors in the input variables follow the same symmetric distribution but the input variable unobserved can be estimated in the proposed QR with EIV. We use the weighted squared loss function in EIV objective function and use the iteratively reweighted least squares procedure to estimate the regression quantiles and input variables unobserved simultaneously.

The rest of this paper is organized as follows. In Section 2 we briefly review the basic principle of orthogonal distance regression. In Section 3 we propose new estimation procedure of ODQR with the weighted squared loss function in EIV objective function. In Section 4 and 5 we present simulation studies and conclusion, respectively.

\section{Orthogonal distance regression}

We first briefly review the basic principle of orthogonal distance regression (ODR). A more detail description can be found in Boggs and Rogers (1990) and some other related references. Then we apply this principle to ODQR in the next section. We first consider the mean regression model with EIV which is defined as follows:

$$
\left\{\begin{array}{l}
y_{i}=f\left(\boldsymbol{x}_{i}^{*}\right)+e_{i}=\boldsymbol{\beta}^{\prime} \boldsymbol{x}_{i}^{*}+b+e_{i} \\
\boldsymbol{x}_{i}=\boldsymbol{x}_{i}^{*}+\boldsymbol{u}_{i}, i=1, \cdots, n,
\end{array}\right.
$$

where $\boldsymbol{x}_{i}^{*}$ is $d \times 1$ vector of unknown and unobservable input vector, $\boldsymbol{u}_{i}$ is $d \times 1$ vector of measurement errors and $\boldsymbol{x}_{i}$ is $d \times 1$ vector of observed input vector. Here, the random errors $e_{i}$ 's are independent and identically distributed with zero mean and finite variance $\sigma^{2}$. The measurement errors $\boldsymbol{u}_{i}$ 's are independent and identically distributed with zero mean and covariance matrix $\Sigma_{u}$. We assume that $e_{i}$ 's and $\boldsymbol{u}_{i}$ 's are uncorrelated. The recent literature has become aware of the inadequacy of assumption that $e_{i}$ 's and $\boldsymbol{u}_{i}$ 's have a joint distribution that is spherically symmetric, and proposes the use of general orthogonal distance regression method that accounts for different uncertainties of the two types of errors. For basic ODR, it is usually assumed that $\Sigma_{u}$ is a diagonal matrix, i.e., $\Sigma_{u}=\sigma^{2} \mathrm{I}_{d \times d}$. For convenience of illustration, we assume that $\boldsymbol{x}_{i}^{*}$ 's are fixed unknown. Then the ODR problem with EIV objective function can be expressed as

$$
L=\sum_{i=1}^{n}\left(y_{i}-\boldsymbol{\beta}^{\prime} \boldsymbol{x}_{i}^{*}-b\right)^{2}+\sum_{i=1}^{n} \sum_{j=1}^{d}\left(x_{i j}-x_{i j}^{*}\right)^{2}
$$

It is proven that this EIV objective function is less prone to bias effects when training with noisy inputs (Van Gorp et al., 2000). However, since both $(\boldsymbol{\beta}, b)$ and $x_{i j}$ 's form the unknown parameters, the parameter space enlarges and thus has $n \times d$ additional parameters. Moreover, since $\boldsymbol{x}_{i}^{*}$ 's in a real world problem are usually not known, these must be estimated 
during the optimization process. Thus, ODR problem (2.2) is rewritten by replacing the above EIV objective function with that without $\boldsymbol{x}_{i}^{*}$ therein such as

$$
L=\sum_{i=1}^{n} \frac{\left(y_{i}-\boldsymbol{\beta}^{\prime} \boldsymbol{x}_{i}-b\right)^{2}}{1+\boldsymbol{\beta}^{\prime} \boldsymbol{\beta}}
$$

In example of $d=1$ the objective function (2.2) and (2.3) are equal to the squared distance between $\left(x_{i}, y_{i}\right)$ and $\left(x_{i}^{*}, \beta x_{i}^{*}+b\right)$, which can be easily seen in Figure 2.1. Squared orthogonal distance between $\left(x_{i}, y_{i}\right)$ and the line $y=\beta x+b$ is the sum of the squared distance between $\left(x_{i}, y_{i}\right)$ and $\left(x_{i}, \beta x_{i}^{*}+b\right)$ and the squared distance between $\left(x_{i}, \beta x_{i}^{*}+b\right)$ and $\left(x_{i}^{*}, \beta x_{i}^{*}+b\right)$, which is $\left(y_{i}-\beta x_{i}^{*}-b\right)^{2}+\left(x_{i}-x_{i}^{*}\right)^{2}$.

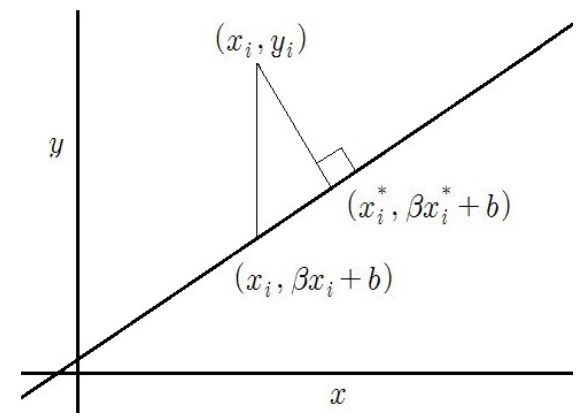

Figure 2.1 Orthogonal distance between $\left(x_{i}, y_{i}\right)$ and the line $y=\beta x+b$

\section{Orthogonal distance quantile regression}

We set $\boldsymbol{x}_{i}^{*}$ is $d \times 1$ vector of unknown and unobservable input vector, $\boldsymbol{u}_{i}$ is $d \times 1$ vector of measurement errors and $\boldsymbol{x}_{i}$ is $d \times 1$ vector of observed input vector. Here, the random errors $e_{i}$ 's and the measurement errors $u_{i j}$ 's are assumed to be independent and identically distributed with zero mean and finite variance $\sigma^{2}$.

He and Liang (2000) proposed the quantile regression in EIV models by using the check function $\rho_{\theta}$ instead of the squared loss function (2.3) as follows:

$$
\min L=\sum_{i=1}^{n} \rho_{\theta}\left(\frac{y_{i}-\boldsymbol{\beta}^{\prime} \boldsymbol{x}_{i}-b}{\sqrt{1+\boldsymbol{\beta}^{2}}}\right),
$$

where $\rho_{\theta}(r)=\theta r I_{(r>0)}+(1-\theta) r I_{(r \leq 0)}$. The objective function (3.1) can be interpreted as the sum of check functions of orthogonal residuals $\left(y_{i}-\boldsymbol{\beta}^{\prime} \boldsymbol{x}_{i}-b\right) / \sqrt{1+\boldsymbol{\beta}^{2}}$, which is also equal to

$$
\begin{aligned}
\sum_{i=1}^{n} & {\left[\theta 1_{\left(y_{i}>\boldsymbol{\beta}^{\prime} \boldsymbol{x}_{i}+b\right)} \times \text { distance between }\left(\boldsymbol{x}_{i}, y_{i}\right) \text { and } y=\boldsymbol{\beta}^{\prime} \boldsymbol{x}_{+} b\right.} \\
& \left.+(1-\theta) 1_{\left(y_{i} \leq \boldsymbol{\beta}^{\prime} \boldsymbol{x}_{i}+b\right)} \times \text { distance between }\left(\boldsymbol{x}_{i}, y_{i}\right) \text { and } y=\boldsymbol{\beta}^{\prime} \boldsymbol{x}_{+} b\right]
\end{aligned}
$$


Here the distance between $\left(\boldsymbol{x}_{i}, y_{i}\right)$ and $y=\boldsymbol{\beta}^{\prime} \boldsymbol{x}+b$ is obtained in (2.2). We proposed the quantile regression in EIV models using (2.2) and (3.2) as follows:

$$
\min L=\sum_{i=1}^{n} W_{i}(\theta)\left[\left(y_{i}-\boldsymbol{\beta}^{\prime} \boldsymbol{x}_{i}^{*}-b\right)^{2}+\sum_{j=1}^{d}\left(x_{i j}-x_{i j}^{*}\right)^{2}\right]
$$

where

$$
\begin{aligned}
W_{i}(\theta) & =\frac{\theta}{\sqrt{\left(y_{i}-\boldsymbol{\beta}^{\prime} \boldsymbol{x}_{i}^{*}-b\right)^{2}+\sum_{j=1}^{d}\left(x_{i j}-x_{i j}^{*}\right)}} 1_{\left(y_{i}>\boldsymbol{\beta}^{\prime} \boldsymbol{x}_{i}+b\right)} \\
& +\frac{1-\theta}{\sqrt{\left(y_{i}-\boldsymbol{\beta}^{\prime} \boldsymbol{x}_{i}^{*}-b\right)^{2}+\sum_{j=1}^{d}\left(x_{i j}-x_{i j}^{*}\right)}} 1_{\left(y_{i} \leq \boldsymbol{\beta}^{\prime} \boldsymbol{x}_{i}+b\right)} .
\end{aligned}
$$

The advantage of the proposed quantile regression in EIV models of (3.3) has advantage over (3.1) that not only the estimates of $(\boldsymbol{\beta}, b)$ but also the estimates of $\boldsymbol{x}_{i}^{*}$ 's can be obtained, which leads to have the estimate of $\boldsymbol{\beta}^{\prime} \boldsymbol{x}_{i}^{*}+b$, where $\boldsymbol{x}_{i}^{*}$ was not observed for training data.

Given $W_{i}(\theta)$ taking partial derivatives of $(3.3)$ with regard to $\left(\boldsymbol{\beta}, b, x_{i j}^{*}\right)$ leads to the optimal values of $\left(\boldsymbol{\beta}, b, x_{i j}^{*}\right)$ to be the solution to

$$
\begin{gathered}
\frac{\partial L}{\partial \boldsymbol{\beta}}=\boldsymbol{x}^{\prime} W(\theta)\left(\boldsymbol{y}-\boldsymbol{\beta}^{\prime} \boldsymbol{x}^{*}-b\right)=\mathbf{0}_{d \times 1}, \frac{\partial L}{\partial \beta_{0}}=\mathbf{1}^{\prime} W(\theta)\left(\boldsymbol{y}-\boldsymbol{\beta}^{\prime} \boldsymbol{x}^{*}-b\right)=0 \\
\frac{\partial L}{\partial x_{i j}^{*}}=W_{i}(\theta)\left(y_{i}-\boldsymbol{\beta}^{\prime} \boldsymbol{x}_{i}^{*}-b\right) \beta_{j}+\left(x_{i j}-x_{i j}^{*}\right)=0, i=1, \cdots, n, j=1, \cdots, d,
\end{gathered}
$$

where $W(\theta)$ is a $n \times n$ diagonal matrix of $W_{i}(\theta)$ 's.

The solution to (3.4) and (3.5) cannot be obtained in a single step since $W_{i}(\theta)$ contains $\left(\boldsymbol{\beta}, b, x_{i j}^{*}\right)$ and is unknown. Thus we need to apply the iteratively reweighted least squares (IRWLS) procedure as follows:

(i) With previous estimates of $x_{i j}^{*}$ and initialized values of $(\boldsymbol{\beta}, b)$

(i-1) Find $W(\theta)$ with $(\boldsymbol{\beta}, b)$.

$(\mathrm{i}-2)$ Find $(\boldsymbol{\beta}, b)$ from $\left(\begin{array}{c}\boldsymbol{\beta} \\ b\end{array}\right)=\left(\begin{array}{cc}\boldsymbol{x}^{* \prime} W(\theta) \boldsymbol{x}^{*} & \boldsymbol{x}^{*} W(\theta) \mathbf{1} \\ \mathbf{1}^{\prime} W(\theta) \boldsymbol{x}^{*} & \mathbf{1}^{\prime} W(\theta) \mathbf{1}\end{array}\right)^{-1}\left(\begin{array}{c}\boldsymbol{x}^{* \prime} W(\theta) \\ \mathbf{1}^{\prime} W(\theta)\end{array}\right) \boldsymbol{y}$.

(i-3) Reiterate above steps until convergence such that $\left\|\boldsymbol{\beta}^{(t)}-\boldsymbol{\beta}^{(t-1)}\right\|^{2}<\mathrm{Tol}_{1}$.

(ii) Find new estimates of $x_{i l}^{*}$ from $(3.5)$ with $(\boldsymbol{\beta}, b)$.

(iii) Reiterate steps (i) and (ii) until convergence such that $\left\|\boldsymbol{x}^{*(t)}-\boldsymbol{x}^{*(t-1)}\right\|^{2}<\mathrm{Tol}_{2}$.

With estimates of $\left(\boldsymbol{\beta}, b, x_{i j}^{*}\right)$ from IRWLS procedure above, the estimator of the $\theta$ th quantile regression function given the input vector $\boldsymbol{x}_{i}^{*}$ (not observed, instead $\boldsymbol{x}_{i}$ was observed for training data) is obtained as follows:

$$
\widehat{q}_{\theta}\left(y \mid \boldsymbol{x}_{i}^{*}\right)=\widehat{\boldsymbol{\beta}}^{\prime} \widehat{\boldsymbol{x}}_{i}^{*}+\widehat{b},
$$


and the estimator of the $\theta$ th quantile regression function given new input vector $\boldsymbol{x}_{t}$ which was not used for the estimation of $\left(\boldsymbol{\beta}, b, x_{i j}^{*}\right)$ is obtained as follows:

$$
\widehat{q}_{\theta}\left(y \mid \boldsymbol{x}_{t}\right)=\widehat{\boldsymbol{\beta}}^{\prime} \boldsymbol{x}_{t}+\widehat{b}
$$

For 1-dimensional input variable we can extend the estimation procedure of ODQR explained above to the polynomial regression case. The squared orthogonal distance between $\left(x_{i}, y_{i}\right)$ and the polynomial curve can be expressed as

$$
\left(y_{i}-f\left(x_{i}^{*}\right)\right)^{2}+\left(x_{i}-x_{i}^{*}\right)^{2}=\frac{\left(y_{i}-f\left(x_{i}^{*}\right)+f^{\prime}\left(x_{i}^{*}\right) x_{i}^{*}-f^{\prime}\left(x_{i}^{*}\right) x_{i}\right)^{2}}{1+f^{\prime}\left(x_{i}^{*}\right)^{2}}
$$

where $f(x)=\sum_{k=1}^{p} \beta_{k} x^{k}+b$ and $\left(x_{i}^{*}, f\left(x_{i}^{*}\right)\right)$ is the closest data point on the polynomial curve to the data point $\left(x_{i}, y_{i}\right)$. In the polynomial regression case with order $2, \boldsymbol{x}^{*}$ in $(3.4)$ is replaced with $\boldsymbol{x}^{*}=\left\{x_{i}^{*}, x_{i}^{* 2}\right\}$ and (3.5) is replaced with

$$
\begin{aligned}
\frac{\partial L}{\partial x_{i}^{*}} & =-f^{\prime}\left(x_{i}^{*}\right)\left(y_{i}-f\left(x_{i}^{*}\right)\right)+x_{i}^{*}-x_{i}=0 \\
& \rightarrow 2 \beta_{2}^{2} x_{i}^{* 3}+3 \beta_{1} \beta_{2} x_{i}^{* 2}+\left(\beta_{1}^{2}-2 \beta_{2} y_{i}+2 b \beta_{2}+1\right) x_{i}^{*}+\left(-\beta_{1} y_{i}+b \beta_{1}-x_{i}\right)=0 .
\end{aligned}
$$

Using (3.9) the estimates of $\left(\boldsymbol{\beta}, b, x_{i}^{*}\right)$ for the polynomial regression are obtained by IRWLS procedure explained above.

\section{Numerical studies}

We illustrate the performance of proposed QR with EIV through the simulated data under different error distributions and quantile levels. We are concerned with the quantile regression in which the input variable is 1-dimensional and 2-dimensional. We use $\theta=0.1$, 0.5, 0.9 for quantile levels. We compare the proposed QR with linear QR. In proposed QR the tolerance $\mathrm{Tol}_{1}$ and $\mathrm{Tol}_{2}$ are set to 0.001 . In the linear $\mathrm{QR}$ the measurement errors in input variables are not considered. We are basically interested in estimating $q_{\theta}\left(\boldsymbol{x}^{*}\right)$ which is the $\theta$ th quantile of $y$ conditional on $\boldsymbol{x}^{*}$ which is the unobserved value of the input vector. Thus, for comparison we calculate the average and standard error of mean squared errors (MSEs) for each estimated quantile regression function as follows:

$$
M S E_{q}=\frac{1}{n} \sum_{i=1}^{n}\left(\widehat{q_{\theta}}\left(y_{i}\right)-q_{\theta}\left(y_{i}\right)\right)^{2}
$$

where $\widehat{q}_{\theta}\left(y_{i}\right)=\widehat{q}_{\theta}\left(\widehat{\boldsymbol{x}}_{i}^{*}\right)$ for the proposed QR, $\widehat{q}_{\theta}\left(y_{i}\right)=\widehat{q}_{\theta}\left(\boldsymbol{x}_{i}\right)$ for the linear QR and $n$ is the size of each data set.We also calculate the average and root mean squared errors (RMSEs) of estimates of regression quantiles as follows:

$$
\operatorname{RMSE}_{\beta}=\sqrt{\frac{1}{n_{t}} \sum_{t=1}^{n_{t}}\left(\widehat{\beta}(\theta)^{(t)}-\beta(\theta)\right)^{2}}
$$


where $\widehat{\beta}(\theta)^{(t)}$ is the estimates of $\beta(\theta)$ (regression quantile; Koenker and Basset, 1978) at $t$ th iteration and $n_{t}$ is the number of iterations. In each example 100 data sets are generated to present the performance of the proposed QR.

Example 4.1 Here $x^{*}$ 's are generated from a uniform distribution $U(0,1)$ and $x$ 's are generated from a normal distribution $N\left(x^{*}, 0.1\right)$. $y$ 's are generated from a normal distribution $N\left(1+2 x^{*}, 0.1\right)$. The true $\theta$ th quantile regression function is given as

$$
q_{0.1}\left(x^{*}\right)=0.5749+2 x^{*}, q_{0.5}\left(x^{*}\right)=1+2 x^{*}, q_{0.9}\left(x^{*}\right)=1.4053+2 x^{*} .
$$

Example 4.2 Here $\left(x_{1}^{*}, x_{2}^{*}\right)$ 's are generated independently from a uniform distribution $U(0,1)$ and $x_{1}$ 's and $x_{2}$ 's are generated from a normal distribution $N\left(x_{1}^{*}, 0.1\right)$ and $N\left(x_{2}^{*}, 0.1\right)$, respectively. $y$ 's are generated from a normal distribution $N\left(1+x_{1}^{*}-2 x_{2}^{*}, 0.1\right)$. The true $\theta$ th quantile regression function is given as

$$
q_{0.1}\left(x_{1}^{*}, x_{2}^{*}\right)=0.5749+x_{1}^{*}-2 x_{2}^{*}, q_{0.5}\left(x_{1}^{*}, x_{2}^{*}\right)=1+x_{1}^{*}-2 x_{2}^{*}, q_{0.9}\left(x_{1}^{*}, x_{2}^{*}\right)=1.4053+x_{1}^{*}-2 x_{2}^{*} .
$$

Example 4.3 Here $x^{*}$ 's and $x$ 's from a uniform distribution $U(0,1)$ and a normal distribution $N\left(x^{*}, 0.1\right)$, respectively. $y$ 's are generated from a normal distribution $N\left(1+0.5 x^{*}+\right.$ $\left.x^{* 2}, 0.25\right)$. The true $\theta$ th quantile regression function is given as

$$
q_{0.1}\left(x^{*}\right)=0.3592+0.5 x^{*}+x^{* 2}, q_{0.5}\left(x^{*}\right)=1+0.5 x^{*}+x^{* 2}, q_{0.9}\left(x^{*}\right)=1.6408+0.5 x^{*}+x^{* 2} .
$$

Figure 4.1 shows true quantile regression functions (solid lines), the estimated quantile regression functions $\widehat{q}_{\theta}\left(x_{i}^{*}\right)$ 's by linear QR (left; dotted lines) and the proposed QR (right; dotted lines), respectively, which are superimposed on the scatter plots of $x$ versus $y$. Here $\widehat{q}_{\theta}\left(x_{i}^{*}\right)$ implies the predicted quantile regression function of response given new input variable $x_{i}^{*}$ based on $100 x$ 's and $100 y$ 's. We can see that quantile regression functions by the proposed $\mathrm{QR}$ are closer to the true quantile regression functions. Table 4.1 shows the average and standard error of $100 \mathrm{MSEs}$ for each estimated quantile regression function. Standard error of MSEs is given in parentheses. As seen from Table 4.1, the proposed QR with EIV yields the smaller averages of MSEs and smaller standard deviations of MSEs for all cases. Table 4.2, 4.3 and 4.4 show the averages and RMSEs for each estimated regression quantiles. From those tables we can see that proposed QR with EIV overal yields the closer estimates of regression quantiles than the linear quantile regression. In tables Boldfaced value indicates the smallest/closest result in each quantile level. From tables we can see that proposed QR with EIV performs better than the linear quantile regression in estimation of quantile regression function and regression quantiles.

Table 4.1 The average of 100 MSEs of estimated quantile regression functions for $\theta=0.1,0.5$ and 0.9 (standard error is in parenthesis)

\begin{tabular}{ccccccc}
\hline \hline \multirow{2}{*}{$\theta$} & \multicolumn{2}{c}{ Ex1 } & \multicolumn{2}{c}{ Ex2 } & \multicolumn{2}{c}{ Ex3 } \\
\cline { 2 - 7 } & LinQR & ODQR & LinQR & ODQR & LinQR & ODQR \\
\hline \multirow{2}{*}{0.1} & 0.2858 & $\mathbf{0 . 1 3 9 0}$ & 0.3549 & $\mathbf{0 . 1 4 9 1}$ & 1.3157 & $\mathbf{0 . 4 1 6 3}$ \\
& $(0.0063)$ & $(0.0030)$ & $(0.0076$ & $(0.0027)$ & $(0.0531)$ & $(0.0156)$ \\
\hline \multirow{2}{*}{0.5} & 0.1950 & $\mathbf{0 . 0 8 1 1}$ & 0.2347 & $\mathbf{0 . 0 8 2 1}$ & 0.8378 & $\mathbf{0 . 2 2 2 0}$ \\
& $(0.0027$ & $(0.0013)$ & $(0.0033)$ & $(0.0014)$ & $(0.0252)$ & $(0.0083)$ \\
\hline \multirow{2}{*}{0.9} & 0.2920 & $\mathbf{0 . 1 3 4 6}$ & 0.3687 & $\mathbf{0 . 1 4 5 2}$ & 1.6599 & $\mathbf{0 . 3 0 3 7}$ \\
& $(0.0077)$ & $(0.0024)$ & $(0.0091)$ & $(0.0028)$ & $(0.0794)$ & $(0.0085)$ \\
\hline
\end{tabular}



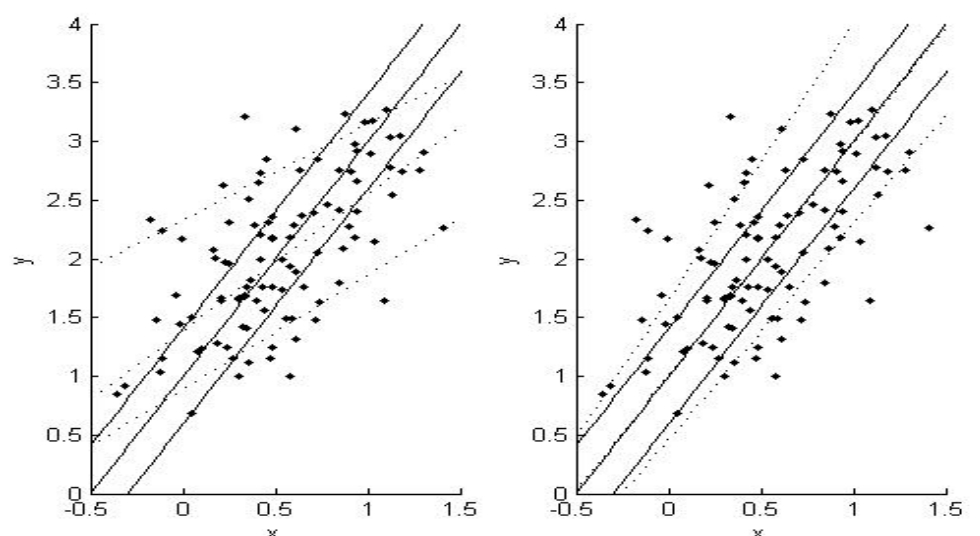

Figure 4.1 True quantile regression functions (solid lines) and the estimated quantile regression functions (dotted lines) superimposed on the scatter plots of and in Example 4.1

Table 4.2 The average of 100 estimates of $b(\theta)$ and $\beta$ for $\theta=0.1,0.5,0.9$ in example 4.1 (RMSE is in parenthesis)

\begin{tabular}{|c|c|c|c|c|c|c|}
\hline \multirow{2}{*}{$\theta$} & \multicolumn{3}{|c|}{$\overline{b(\theta)}$} & \multicolumn{3}{|c|}{$\bar{\beta}$} \\
\hline & true & $\overline{L i n Q R}$ & ODQR & true & LinQR & ODQR \\
\hline 0.1 & 0.5749 & $\begin{array}{c}\mathbf{0 . 8 7 9 7} \\
(0.3137)\end{array}$ & $\begin{array}{c}0.2295 \\
(0.5343)\end{array}$ & 2 & $\begin{array}{c}0.8576 \\
(1.1641)\end{array}$ & $\begin{array}{l}\mathbf{1 . 9 4 0 9} \\
(0.4208\end{array}$ \\
\hline 0.5 & 1 & $\begin{array}{c}1.5450 \\
(0.5554)\end{array}$ & $\begin{array}{c}\mathbf{1 . 0 0 6 0} \\
(0.1811)\end{array}$ & 2 & $\begin{array}{c}0.9221 \\
(1.0881)\end{array}$ & $\begin{array}{l}\mathbf{1 . 9 7 6 6} \\
(0.3119)\end{array}$ \\
\hline 0.9 & 1.4053 & $\begin{array}{c}2.2949 \\
(0.9050)\end{array}$ & $\begin{array}{c}\mathbf{1 . 8 0 5 7} \\
(0.4181)\end{array}$ & 2 & $\begin{array}{c}0.8180 \\
(1.2016)\end{array}$ & $\begin{array}{l}\mathbf{1 . 9 0 4 8} \\
(0.3950)\end{array}$ \\
\hline
\end{tabular}

Table 4.3 The average of 100 estimates of $b(\theta), \beta_{1}$ and $\beta_{2}$ for $\theta=0.1,0.5,0.9$ in example 4.2 (RMSE is in parenthesis)

\begin{tabular}{|c|c|c|c|c|c|c|c|c|c|}
\hline \multirow{2}{*}{$\theta$} & \multicolumn{3}{|c|}{$\overline{b(\theta)}$} & \multicolumn{3}{|c|}{$\beta_{1}$} & \multicolumn{3}{|c|}{$\beta_{2}$} \\
\hline & true & LinQR & ODQR & true & LinQR & ODQR & true & LinQR & ODQR \\
\hline 0.1 & 0.5749 & $\begin{array}{c}-0.0204 \\
(0.6505)\end{array}$ & $\begin{array}{c}\mathbf{0 . 1 0 9 0} \\
(0.6439)\end{array}$ & -2 & $\begin{array}{c}-0.8729 \\
(1.1521)\end{array}$ & $\begin{array}{l}\mathbf{- 1 . 9 1 3 4} \\
(0.4306)\end{array}$ & 1 & $\begin{array}{c}0.4531 \\
(0.6024)\end{array}$ & $\begin{array}{r}\mathbf{0 . 9 6 2 7} \\
(0.5378)\end{array}$ \\
\hline 0.5 & 1 & $\begin{array}{c}0.7430 \\
(0.2891)\end{array}$ & $\begin{array}{c}\mathbf{0 . 9 9 8 9} \\
(0.3003) \\
\end{array}$ & -2 & $\begin{array}{c}-0.9436 \\
(1.0653) \\
\end{array}$ & $\begin{array}{c}\mathbf{- 1 . 9 2 7 9} \\
(0.3137)\end{array}$ & 1 & $\begin{array}{c}0.4677 \\
(0.5557) \\
\end{array}$ & $\begin{array}{r}\mathbf{0 . 9 4 3 8} \\
(0.4048) \\
\end{array}$ \\
\hline 0.9 & 1.4053 & $\begin{array}{c}\mathbf{1 . 4 4 3 6} \\
(0.1997) \\
\end{array}$ & $\begin{array}{c}1.8344 \\
(0.6362) \\
\end{array}$ & -2 & $\begin{array}{c}-0.8516 \\
(1.1724) \\
\end{array}$ & $\begin{array}{r}\mathbf{- 1 . 8 7 9 8} \\
(0.4609)\end{array}$ & 1 & $\begin{array}{c}0.4645 \\
(0.5942)\end{array}$ & $\begin{array}{r}\mathbf{0 . 9 5 4 8} \\
(0.5381)\end{array}$ \\
\hline
\end{tabular}

Table 4.4 The average of 100 estimates of $b(\theta), \beta_{1}$ and $\beta_{2}$ for $\theta=0.1,0.5,0.9$ in example 4.3 (RMSE is in parenthesis)

\begin{tabular}{|c|c|c|c|c|c|c|c|c|c|}
\hline \multirow{2}{*}{$\theta$} & \multicolumn{3}{|c|}{$\overline{b(\theta)}$} & \multicolumn{3}{|c|}{$\bar{\beta} \beta_{1}$} & \multicolumn{3}{|c|}{$\beta_{2}$} \\
\hline & true & LinQR & ODQR & true & LinQR & ODQR & true & LinQR & ODQR \\
\hline 0.1 & 0.3592 & $\begin{array}{c}0.4186 \\
(0.1556)\end{array}$ & $\begin{array}{c}\mathbf{0 . 3 7 2 8} \\
(0.2377)\end{array}$ & 0.5 & $\begin{array}{c}0.2340 \\
(0.2992)\end{array}$ & $\begin{array}{c}\mathbf{0 . 4 9 7 1} \\
(0.2558)\end{array}$ & 1 & $\begin{array}{c}0.4139 \\
(0.6001)\end{array}$ & $\begin{array}{r}\mathbf{1 . 0 0 3 6} \\
(0.4481) \\
\end{array}$ \\
\hline 0.5 & 1 & $\begin{array}{l}\mathbf{1 . 1 4 0 5} \\
(0.1895)\end{array}$ & $\begin{array}{c}0.7872 \\
(0.2735)\end{array}$ & 0.5 & $\begin{array}{c}0.3820 \\
(0.1957)\end{array}$ & $\begin{array}{c}\mathbf{0 . 4 4 2 3} \\
(0.2057)\end{array}$ & 1 & $\begin{array}{c}0.6332 \\
(0.3855)\end{array}$ & $\begin{array}{r}\mathbf{0 . 9 9 9 6} \\
(0.1777)\end{array}$ \\
\hline 0.9 & 1.6408 & $\begin{array}{c}2.0886 \\
(0.4876)\end{array}$ & $\begin{array}{c}\mathbf{1 . 6 3 5 2} \\
(0.3359)\end{array}$ & 0.5 & $\begin{array}{c}0.5824 \\
(0.2872)\end{array}$ & $\begin{array}{c}\mathbf{0 . 5 1 1 5} \\
(0.4805)\end{array}$ & 1 & $\begin{array}{c}0.9561 \\
(0.1952)\end{array}$ & $\begin{array}{r}\mathbf{0 . 9 9 5 5} \\
(0.4220)\end{array}$ \\
\hline
\end{tabular}




\section{Conclusions}

In this paper, we dealt with estimating regression quantiles and quantile regression function in EIV models by using the weighted squared loss function in EIV objective function. We found that the proposed QR provides satisfying results in estimating regression quantiles and quantile regression functions in the given examples. The proposed QR can be extended to the nonlinear quantile regression in EIV models by introducing support vector machine (Vapnik, 1998) and support vector quantile regression (Takeuchi et al., 2006), which can be applied easily and effectively to the nonlinear quantile regression in EIV models and the high dimensional input vector.

\section{References}

Boggs, P. T. and Rogers, J. E. (1990). Orthogonal distance regression. Contemporary Mathematics, 112, 183-194.

Boggs, P. T., Byrd, R. H. and Schnabel, R. B. (1987). A stable and efficient algorithm for nonlinear orthogonal distance regression. SIAM Journal on Scientific Computing, 8, 105-1078.

Chesher, A. (2001). Parameter approximations for quantile regressions with measurement error, Working Paper CWP02/01, Department of Economics, University College London, London, UK.

Fuller, W. A. (1987). Measurement error models, Wiley, New York.

$\mathrm{He}$, X. and Liang, H. (2000). Quantile regression estimates for a class of linear and partially linear errorsin-variables models. Statistica Sinica, 10, 129-140.

$\mathrm{Hu}$, Y. and Schennach, S. M. (2008). Identification and estimation of nonclassical nonlinear errors-invariables models with continuous distributions using instruments. Econometrica, 76, 195-216.

Hwang, C. (2010). M-quantile regression using kernel machine technique. Journal of the Korean Data $\mathcal{E}$ Information Science Society, 21, 973-981.

Ioannidesa, D. A. and Matzner-L $\phi$ ber, E. (2009). Regression quantiles with errors-invariables. Journal of Nonparametric Statistics, 21, 1003-1015.

Koenker, R. (2005). Quantile regression, Cambridge University Press, Cambridge.

Koenker, R. and Bassett, G. (1978). Regression quantile. Econometrica, 46, 33-50.

Koenker, R., Ng, P. and Portnoy, S. (1994). Quantile smoothing splines, Biometrika, 81, 673-680.

Lee, S. (2012). Forecasting value-at-risk by encompassing CAViaR models via information criteria. Journal of the Korean Data \& Information Science Society, 24, 1531-1541.

Ma, Y. and Yin, G. (2011). Censored quantile regression with covariate measurement errors. Statistica Sinica, 21, 949-971.

Schennach, S. M. (2008). Quantile regression with mismeasured covariates. Econometric Theory, 24, 10101043.

Shim, J. and Lee, J. (2010). Restricted support vector regression without crossing. Journal of the Korean Data 83 Information Science Society, 21, 1319-1325.

Takeuchi, I., Le, Q. V., Sears, T. and Smola, A. J. (2006). Nonparametric quantile regression. Journal of Machine Learning Research, 7, 1231-1264.

Van Gorp, J., Schoukens, J. and Pintelon, R. (2000). Learning neural networks with noisy inputs using the errors-in-variables approach. IEEE Transactions on Neural Networks, 11, 402-414.

Vapnik, V. N. (1998). Statistical learning theory, John Wiley, New York.

Wang, H. J., Stefanski, L. A. and Zhu, Z. (2012). Corrected-loss estimation for quantile regression with covariate measurement errors. Biometrika, 99, 405-421.

Wei, Y. and Carroll, R. J. (2009). Quantile regression with measurement error. Journal of the American Statistical Association, 104, 1129-1143.

Yu, K., Lu, Z. and Stander, J. (2003). Quantile regression: Applications and current research area. The Statistician, 52, 331-350. 